\title{
Complicaciones de la prostatectomía radical: evolución y manejo conservador de la incontinencia urinaria
}

\author{
Juan Escudero JU, Ramos de Campos M, Ordoño Domínguez F, Fabuel Deltoro M, \\ Navalón Verdejo P, Zaragozá Orts J.
}

Servicio de Urología. Consorcio Hospital General Universitario de Valencia. Valencia.

Actas Urol Esp. 2006;30(10):991-997

\section{RESUMEN \\ COMPLICACIONES DE LA PROSTATECTOMÍA RADICAL: EVOLUCIÓN Y MANEJO CONSERVADOR DE LA INCONTINENCIA URINARIA}

Objetivos: La prostatectomía radical retropúbica continúa siendo el tratamiento de elección para el cáncer de próstata órganoconfinado. Realizamos un estudio retrospectivo en el que valoramos las complicaciones de esta cirugía y su evolución en el tiempo, así como la utilidad del tratamiento conservador, especialmente la rehabilitación perineal precoz, en la recuperación de la continencia tras esta intervención.

Material y métodos: Durante el periodo comprendido entre diciembre de 1998 a enero del 2005 hemos intervenido en nuestro servicio a 168 pacientes afectos de adenocarcinoma prostático confinado clínicamente a la glándula mediante prostatectomía radical retropúbica. Retiramos la sonda vesical el decimocuarto día del postoperatorio. A los pacientes que presentaron incontinencia urinaria (IU) de esfuerzo se les instruyó para la realización de ejercicios para fortalecer el suelo pélvico. Realizamos un seguimiento clínico el primer, tercer y sexto mes tras la cirugía, y posteriormente de forma semestral.

Resultados: De los 168 pacientes intervenidos, el 15\% requirió hemotransfusión en el postoperatorio inmediato. Otras complicaciones precoces, de mucha menor incidencia, fueron la perforación intestinal, la infección de la herida quirúrgica y la formación de hematoma en el lecho quirúrgico. Entre las complicaciones tardías más frecuentes registramos la disfunción eréctil, que se presentó en 164 pacientes (98\%), alteraciones miccionales en 32 pacientes (19\%) y alteraciones intestinales en aproximadamente el $11 \%$. Entre las alteraciones miccionales encontradas, la más frecuente fue la IU (64\%) si bien la mayoría de ellas eran de carácter leve (34\%) y todas mejoraron durante los primeros seis meses posteriores a la cirugía.

Conclusiones: La IU es el principal condicionante de la calidad de vida de los pacientes que son sometidos a prostatectomía radical. Si bien el porcentaje de pacientes que la presenta es alto, la mayoría son de grado leve o moderado y pueden mejorar ostensiblemente con los ejercicios de fortalecimiento del suelo pélvico, constituyéndose así como un arma fundamental en el manejo de estos pacientes, acompañada o no del uso de absorbentes, de la terapia farmacológica o del empleo de colectores peneanos.

Palabras clave: Prostatectomía radical retropúbica. Complicaciones quirúrgicas. Incontinencia urinaria.

\begin{abstract}
RADICAL PROSTATECTOMY COMPLICATIONS: EVOLUTION AND CONSERVATIVE MANAGEMENT OF URINARY INCONTINENCE

Objectives: Radical retropubic prostatectomy is the major procedure for curing early stage prostate cancer. In this retrospective study we valued the complications of this surgery and its evolution in time, as well as the efectiveness of the urinary incontinence preservative treatment in the recovery of urinary continence.

Materials and methods: During the period between december of 1998 to january of the 2005 we have treated 168 patients suffering prostatic adenocarcinoma clinically confined to the gland by means of retropubic radical prostatectomy. We removed the vesical catheter the fourteenth day of the postoperative and made a clinical monitoring the first, third and sixth month after the surgery, and thereafter every six months.

Results. Of the 168 patients who underwent clinical surgery, 15\% required postoperative blood transfusion in the immediate postoperative. Other complications, with a lower incidence, were the intestinal perforation, surgical wound infection and the haematoma.

The most frequent delayed complications we registered were erectile dysfunction, observed in 164 patients (98\%), micturition alterations in 32 patients (19\%) and intestinal alterations in approximately $11 \%$. Among the micturitional alterations found, the most frequent (64\%) was the Urinary Incontinence (UI). However, most of them were of minor importance (34\%) and all improved during the first six months after the surgery. We advised patients with minor and moderate UI to perform pelvic floor muscle training, being very useful since they allowed to anticipate the recovery of continence.

Conclusions. Radical prostatectomy continues to be the most effective treatment for the non spared prostate cancer, in spite of the associated morbidity. The IU is the main factor for the high standard of living these patients. Despite the number of patients with UI being so high, most cases are moderate and minor and may improve with pelvic floor muscle training. Hence, this is a significant tool to tackle this illness, whether in connection with the use of absorbent, the pharmacologic therapy or the use of penile collectors.
\end{abstract}

Keywords: Radical retropubic prostatectomy. Surgical injuries. Urinary incontinence. 
$\mathrm{T}$ ras 50 años de la aparición de la prostatectomía radical perineal de Young, Millin describió el acceso retropúbico, y, a pesar de la aparición de nuevas técnicas quirúrgicas y no estar exenta de complicaciones, la prostatectomía radical retropúbica con linfadenectomía obturatriz, continúa siendo la técnica quirúrgica de elección para el tratamiento del cáncer de próstata órgano-confinado.

Entre las complicaciones precoces más frecuentes se encuentran la anemización aguda por sangrado peroperatorio, la formación de urinomas y linfoceles, la lesión rectal, la neuroapraxia y la infección de la herida quirúrgica. De las complicaciones a medio y largo plazo, las más frecuentes son la disfunción eréctil y las alteraciones miccionales, siendo éstas últimas las que ocasionan una mayor afectación en la calidad de vida de los pacientes. Si bien el número de pacientes que presenta incontinencia tras la cirugía radical es alto, muchas de estas incontinencias mejoran durante los seis primeros meses, pero, a pesar de esto, es necesario instaurar un tratamiento que mejore la sintomatología mientras esto sucede y que anticipe la recuperación.

\section{MATERIAL Y MÉTODOS}

Entre diciembre de 1998 y enero de 2006 hemos intervenido en nuestro centro a 168 pacientes con diagnóstico de adenocarcinoma de próstata clínicamente confinado a la glándula (92 con estadio clínico T1NOMO y 76 con T2NOMO). La media de edad de los pacientes intervenidos fue de 63 años, con un rango comprendido entre los 42 y los 76 años. Para alcanzar el diagnóstico, a todos ellos se les realizó: tacto rectal, determinación del PSA y biopsia prostática transrectal ecodirigida, así como estudio de extensión consistente en una gammagrafía ósea y resonancia magnética endocavitaria multiantena. $\mathrm{El}$ tacto rectal fue sugestivo de neoformación en 95 de los 168 pacientes $(56,55 \%)$ y de HBP en $73(43,45 \%)$. El PSA medio fue de $12,47 \mathrm{ng} / \mathrm{ml}$ con un rango de 1,9 a $19 \mathrm{ng} / \mathrm{mL}$. En todos ellos el rastreo óseo fue negativo. El Gleason preoperatorio fue de grado bajo en 88 pacientes $(52,4 \%)$, medio en 74 $(44,1 \%)$ y alto en $6(3,6 \%)$.

A todos los pacientes se les practicó una prostatectomía radical retropúbica con linfadenecto- mía obturatriz bilateral extemporánea en los casos con Gleason mayor o igual a 8 o PSA mayor de $10 \mathrm{ng} / \mathrm{ml}$. No se llevó a cabo preservación de bandeletas neurovasculares, realizando cuidadosa disección apical con el fin de preservar tanta uretra sana como fuera posible, asímismo en todos los pacientes se procuró la preservación del cuello vesical, y se realizó la anastomosis cervicouretral con cinco puntos de material de reabsorción rápida y eversión de bordes cervicales. La sonda vesical fue retirada el decimocuarto día del postoperatorio en la mayoría de los casos, salvo en aquellos que presentaron retención aguda de orina en el momento de la retirada. Tras el alta hospitalaria y la retirada de la sonda, se realizó un seguimiento clínico al primer, tercer y sexto mes de la intervención y, posteriormente, las revisiones se espaciaron semestralmente. En dichos seguimientos, se preguntó a los pacientes sobre la sintomatología miccional, tanto incontinente como obstructiva, así como por la función sexual y la posible sintomatología irritativa rectal, teniendo en cuenta sus variaciones respecto a la situación previa a la cirugía.

Clasificamos la incontinencia urinaria en diferentes niveles de intensidad, siendo: a) leve, ante control miccional con algún escape, no siendo necesario el uso de absorbentes; b) moderada, si el paciente presenta escapes miccionales ocasionales con uso de no más de un absorbente diario, y c) severa, ante escapes miccionales frecuentes o totales, precisando el uso de varios absorbentes al cabo del día.

A los pacientes en los que encontramos cualquier grado de incontinencia les realizamos medición del residuo postmiccional por medio de ecografía transabdominal para descartar obstrucción concomitante que produjese micción por rebosamiento.

Al alta hospitalaria, los pacientes fueron instruidos para realizar ejercicios del suelo pélvico, cuya finalidad es conseguir una adecuada transmisión de presiones para alcanzar una estática pélvica óptima, y además, fortalecer el esfinter estriado que permite la continencia durante el esfuerzo. Para la realización de dichos ejercicios el paciente debe previamente identificar el grupo muscular de trabajo, para ello el paciente realiza interrupciones del chorro miccional evitando la 
contracción de los glúteos, aductores y rectos, en una primera sesión tutorizada por el clínico y en su domicilio más tarde. Posteriormente, y siempre en reposo miccional, el paciente realiza una serie de ejercicios consistentes en 10 contracciones lentas y mantenidas intercaladas con 10 rápidas durante un tiempo no inferior a $10 \mathrm{minu}-$ tos cada día, 2 a 5 veces por semana durante 12 semanas.

\section{RESULTADOS}

De los 168 pacientes intervenidos, 56 (33,4\%) presentaron márgenes afectos en el estudio anatomopatológico precisando completar el tratamiento con la administración de radioterapia externa adyuvante en el lecho quirúrgico y en las cadenas ganglionares pélvicas. A $50(29,7 \%)$ de los 168 pacientes se les trató con hormonoterapia por fracaso bioquímico del PSA, administrándoles análogos de LH-RH humana asociados a antiandrógenos de este último grupo 23 habían recibido radioterapia adyuvante tras despistaje de presencia de metástasis al presentar progresión bioquímica.

El seguimiento medio fue de 26,6 meses, con un rango que ha oscilado entre las 16 y las 250 semanas.

Entre las complicaciones precoces recogidas, la más frecuente fue el sangrado peroperatorio, que requirió hemotransfusión en 25 casos $(14,8 \%)$, administrándose entre 1 y 6 concentrados de hematíes (media 1,9). La infección de la herida quirúrgica aconteció en 11 pacientes (6,5\%); en 8 casos hubo perforación intestinal $(4,76 \%)$, y 4 pacientes $(2,4 \%)$ presentaron hematoma en el lecho quirúrgico, precisando tan sólo 1 paciente la reintervención para reubicar la sonda vesical. La neuroapraxia del obturador fue una complicación observada en 6 pacientes, resuelta en todos los casos en los primeros tres meses tras la cirugía. En nuestra serie no registramos ningún caso de infarto agudo de miocardio, accidentes cerebrovasculares, tromboembolimo pulmonar, ni de trombosis venosa profunda o fistula urinaria o rectal.

De las complicaciones a medio y largo plazo, cabe destacar la disfunción eréctil, que se presentó en 164 pacientes $(97,6 \%)$. Ésta fue definida como disminución de la líbido, de la frecuencia de la actividad sexual o de la calidad y duración de la erección en pacientes que previamente a la cirugía no presentaban impotencia. Constatamos alteraciones intestinales en 22 pacientes, referidas como diarrea, urgencia o incontinencia fecal, pero también como tenesmo rectal o congestión anal; que desaparecieron en su totalidad antes del cuarto mes del postoperatorio. Encontramos alteraciones miccionales en cerca del $20 \%$ de todos los pacientes intervenidos. La retención secundaria a estenosis de la anastomosis vesicouretral, descrita en otras series de la literatura, no fue encontrada en ninguno de nuestros pacientes, predominando la incontinencia urinaria de esfuerzo, siendo predominantemente de carácter leve en todo momento.

En revisiones sucesivas, tras la realización de los ejercicios de fortalecimiento del suelo pélvico, se aprecia una ostensible mejoría de la sintomatología. Inmediatamente después de retirar la sonda vesical (Fig. 1) pudimos constatar que un $64 \%$ de pacientes presentaban diferentes grados de incontinencia, la mayoría de ellos de carácter leve. Tras la primera revisión, registramos una amplia disminución en el número de casos afectos de incontinencia severa, mientras los casos de incontinencia leve y moderada aumentaron a expensas de aquellos con IU severa y moderada respectivamente, por su mejoría. En sucesivas revisiones se mantuvo esta tendencia, si bien el tiempo en presentar dicha mejoría aumentó considerablemente. En la Figura 1 podemos apreciar

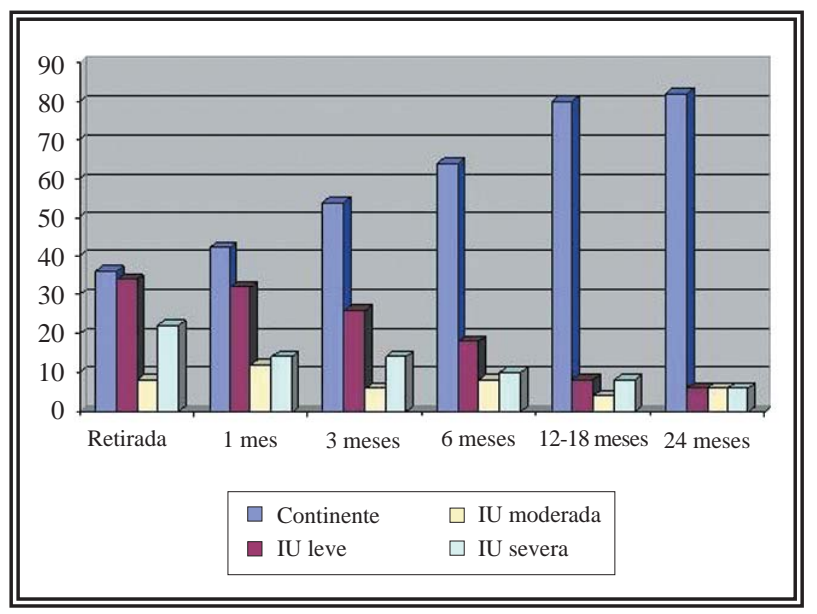

FIGURA 1. Relación porcentual de pacientes con IU según su grado tras la retirada de la sonda y en las revisiones sucesivas. 
también como desciende significativamente el porcentaje de pacientes con IU moderada y severa, que presentan clara mejoría, para terminar alcanzando un $18 \%$ del total de individuos incluidos en el estudio. Finalmente, el $71 \%$ de pacientes que en un principio presentaron IU de cualquier grado alcanzaron la continencia, y tan sólo el $12 \%$ de los intervenidos mantuvieron grados moderado o severo de IU. El $82 \%$ del total de pacientes intervenidos eran continentes a los 24 meses, con un tiempo medio de recuperación de 6,9 meses. Un $6 \%$ presentaban IU leve, un $6 \%$ moderada y otro $6 \%$ severa. $18(11 \%)$ pacientes no completaron las 12 semanas de tratamiento prescrito, de los cuales 14 (78\%) presentaban incontinencia moderada y severa en el momento en que abandonaron el tratamiento.

Tan sólo 7 pacientes presentaron retención de orina, secundaria a hematuria con coágulos que fueron tratadas con sondaje vesical y lavados vesicales activos, siendo posible la retirada del mismo en todos ellos antes del mes postoperatorio. Un $50 \%$ de los pacientes intervenidos presentaron en algún momento del postoperatorio cierto grado de inestabilidad vesical que mejoró ostensiblemente con farmacoterapia anticolinérgica.

En nuestra serie, y en series revisadas en la literatura ${ }^{10,17}$, encontramos que el tiempo en alcanzar la continencia en los varones de menor edad era menor. En nuestra serie, el 72\% de los pacientes que pasaron de presentar cualquier grado de incontinencia a recuperar la continencia total eran menores de 65 años. Esto se puede explicar por la distrofia que sufre el esfinter estriado uretral con la edad. No encontramos diferencias estadísticamente significativas en el tiempo de recuperación de la continencia entre los pacientes irradiados y el resto de casos.

\section{DISCUSIÓN}

La continencia urinaria, conjuntamente con la potencia sexual, son los aspectos que más preocupan al paciente afecto de cáncer de próstata órgano-confinado al que se le plantea la opción de tratamiento quirúrgico radical. Hemos definido la continencia, en general, como la ausencia de escapes miccionales involuntarios y de uso de absorbentes miccionales.
La incidencia de IU en todos sus grados varía entre 0 y $20 \%$ según las series dependiendo sobre todo de los criterios utilizados para definirla, los métodos usados para cuantificarla y de la experiencia del centro en realizar esta técnica quirúrgica. Diferentes trabajos han puesto de manifiesto la importancia de los criterios utilizados para medir la incontinencia a la hora de valorar su incidencia. Si bien estos datos no han sido sujetos a un riguroso estudio estadístico ${ }^{1}$, parecen demostrar que existen diferencias en cuanto al tiempo de recuperación de la continencia, dependiendo de si ésta se recoge de lo reportado por el paciente o de lo inquirido por el urólogo ${ }^{2}$.

La cirugía radical prostática disminuye la longitud anatómica y funcional uretral, su elasticidad y su vascularización e inervación. La preservación de tanta uretra como sea posible durante la disección apical de la próstata es muy importante a la hora de conseguir una buena continencia urinaria. Coakley et al. ${ }^{6}$ describieron la asociación entre un menor tiempo de recuperación de la continencia y la mayor longitud de uretra membranosa preservada durante la cirugia. En este mismo, sentido Gaker et al. ${ }^{7}$ describen una técnica de conservación de parte de la uretra prostática, que no sólo ayuda a una más rápida recuperación de la continencia y a una mayor tasa de pacientes continentes, si no a un porcentaje menor de estenosis de la anastomosis uretrovesical. Como se ha comentado anteriormente, también se ha descrito la importancia de la conservación quirúrgica del cuello vesical, reportando porcentajes de recuperación de la continencia de hasta el $89 \%$ con el uso de esta técnica ${ }^{8}$, si bien no hay consenso en la literatura sobre la capacidad de esta técnica para mejorar la recuperación y alcanzar la continencia comparada con la reconstrucción del cuello vesical ${ }^{9}$.

$\mathrm{O}^{\text {`Donell y Walsh }}{ }^{5}$ describieron la importancia de la conservación de las bandeletas neurovasculares a la hora de alcanzar la continencia tras la cirugía radical, también la preservación del cuello vesical, la tubulización del mismo y su remodelación han sido propuestos como técnicas que ofrecen mejor resultado en cuanto a la preservación de la continencia; pero la radicalidad necesaria para esta cirugía y la anatomía tumoral, en muchas ocasiones, hacen que llevar esto a cabo 
sea imposible. Las técnicas de conservación de bandeletas neurovasculares aportan en algunas series tasas de recuperación de la continencia de hasta el 93\% a partir de los 18 meses de seguimiento, encontrando diferencias estadísticamente significativas en cuanto a la recuperación de dicha continencia estratificando el estudio por edades, a favor de los pacientes en la cuarta y quinta década de la vida ${ }^{4,5}$.

En la literatura revisada parece existir consenso en cuanto a que el tratamiento de la incontinencia de esfuerzo postprostatectomía radical sea conservador durante los primeros 9 a 12 meses. Entre los tratamiento conservadores encontramos la cinesiterapia, la electroestimulación y el biofeedback. A pesar de lo extendido de estos tratamientos no existe evidencia de que alternativas como el biofeedback o estimulación eléctrica sean superiores a los ejercicios del suelo pélvico realizados aisladamente para reducir el tiempo en alcanzar la continencia además añaden al tratamiento un coste adicional $^{17}$. A esto hay que añadir la gran ventaja de que la fisioterapia del suelo pélvico no tenga prácticamente efectos secundarios y ofrezca la posibilidad de combinarla con tratamiento farmacológico en casos de incontinencia urinaria mixta.

Aunque las tasas finales de continencia a partir de los 18 meses no varían de forma estadísticamente significativa en todos los estudios que comparan grupos tratados con fisioterapia del suelo pélvico con grupos control ${ }^{10,16}$, si que coinciden en que existen diferencias en cuanto al tiempo transcurrido en recuperar la continencia. En este sentido, Filocamo et al. ${ }^{10}$ reportan una tasa de continencia del 19\% y del 95\% al mes y a los seis meses, respectivamente en los pacientes que realizaron ejercicios del suelo pélvico, frente al 8\% y el 65\% en los pacientes del grupo control (Fig. 2). Cornel et al. ${ }^{11}$ También estudiaron el efecto de la reeducación del suelo pélvico realizada tempranamente sobre el tiempo de recuperación de la continencia, encontrando un 40\% de pacientes continentes al primer mes, 70\% al segundo, un $86 \%$ a los 6 meses y un $88 \%$ al año, y concluyen, al comparar sus resultados con los de la literatura, que el tiempo hasta alcanzar la continencia en los pacientes prostatectomizados es sensiblemente menor si comienzan a realizar ejercicios del suelo pélvico desde el momento de la retirada del catéter.

La Sociedad Internacional de Continencia define la incontinencia urinaria como la pérdida involuntaria de orina que condiciona un problema higiénico y/o social. A pesar de que esta definición es empleada por la mayoría de los autores, no existe consenso a la hora de cuantificar el grado de dicha patología. Así Filocamo et al. ${ }^{10}$ consideran continentes a aquellos pacientes que emplean un absorbente diario, mientras que en nuestra serie estos pacientes serían considerados como incontinentes. Por otro lado, Cornel et al. ${ }^{11}$ consideran continentes a aquellos pacientes con pérdidas miccionales inferiores a $2 \mathrm{~g}$ en los absorbentes o bien la ausencia del empleo de estos; este grupo de pacientes en nuestra serie sería catalogado como incontinencia leve o moderada. Por este motivo, los resultados de las series mencionadas en este artículo no son comparables (Fig. 2), no existiendo un baremo unificado para establecer la severidad de la incontinencia, siendo los pacientes que nosotros clasificamos como incontinentes leves y gran parte de los incontinentes moderados considerados en otras series como continentes.

Como hemos apuntado, no está claro que los ejercicios del suelo pélvico mejoren las tasas finales de continencia de estos pacientes ${ }^{10,15}$, por lo que en caso de fracasar podemos utilizar otras medidas para el tratamiento de la incontinencia. Entre las medidas conservadoras disponemos del uso de sistemas de oclusión externa uretral que, si bien reducen el número de absorbentes y la cantidad de escape intermiccional, pensamos que resultan incómodos y en ocasiones insuficientes cuando se eleva la presión intraabdominal, quedando limitado su uso casi exclusivamente para incontinencias de carácter leve cuando el paciente no desea ser intervenido nuevamente. Igualmente, en esta línea de tratamiento conservador podemos utilizar sistemas colectores peneanos y absorbentes, dependiendo del grado de incontinencia. En cuanto al tratamiento médico, se han utilizado sustancias alfa agonistas, capaces de incrementar el tono cervical, como la efedrina o la imipramina con resultados equívocos, pero su uso parece tener una eficacia muy limitada en el tratamiento de la incontinencia postprostatectomía. 


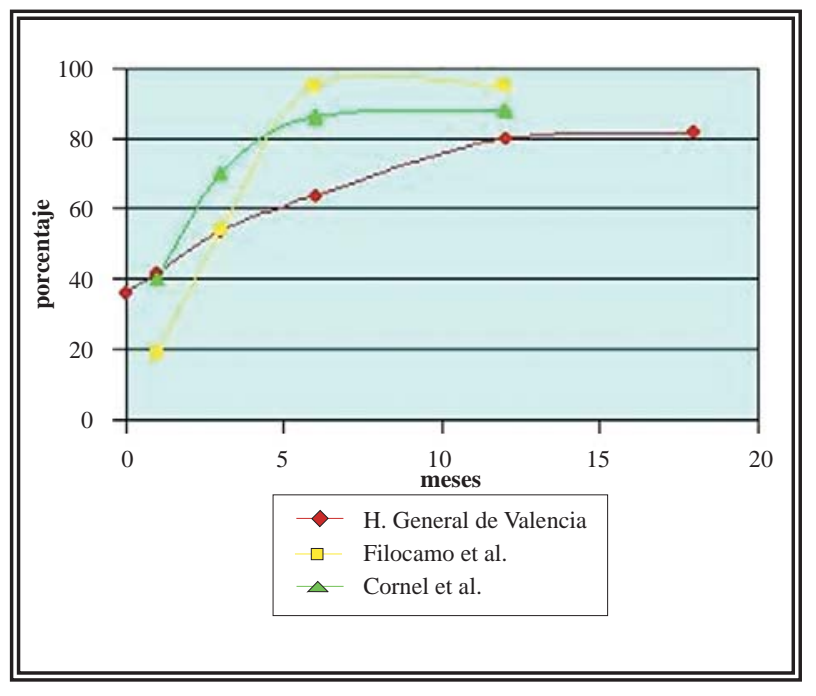

FIGURA 2. Tiempo en recuperar la continencia.

Dentro de las alternativas terapéuticas invasivas, se encuentra la inyección de las sustancias ocupantes de espacio, sin embargo, su uso está limitado en varones intervenidos de esta patología por aspectos técnicos, con tasa de éxito que han variado entre el 36 y el 69\%, tras normalmente numerosas inyecciones y no están recomendados por las guías de la Asociación Europea de Urologia.

Otra alternativa de tratamiento invasivo para casos de incontinencia moderada, cuyo uso actualmente más se está extendiendo, es el empleo de las mallas suburetrales de polipropileno o silicona, fijas o reajustables, que han presentado resultados satisfactorios y que entre sus ventajas cuenta con la fácil ejecución técnica, el mantenimiento de una micción fisiológica y una buena tolerancia por parte del paciente ${ }^{12}$. En los últimos años se ha introducido el uso de cabestrillos suburetrales reajustables con óptimos resultados y escasas complicaciones a corto plazo $^{14}$.

Finalmente, el esfinter urinario artificial parece el tratamiento más eficaz a largo plazo para la incontinencia de esfuerzo severa secundaria a la cirugía radical prostática, ha demostrado buenos resultados en series de hasta 10 años de seguimiento, siendo una técnica segura y eficaz, que llega a tasas de continencia y satisfacción que alcanzan el $90 \%{ }^{13}$, si bien no es despreciable el elevado número de reintervenciones, en ocasiones para la retirada de la prótesis por erosión o atrofia uretral, además de no proporcionar una micción fisiológica ya que requiere la activación de un dispositivo mecánico.

\section{CONCLUSIONES}

La incontinencia urinaria continúa siendo una de las complicaciones más frecuentes a corto y medio plazo de la prostatectomía radical, constituyendo una secuela que disminuye considerablemente la calidad de vida de estos pacientes. Aunque diversos factores están implicados en la tasa final de IU, la técnica quirúrgica parece ser el más importante presentando menor morbilidad los pacientes sometidos a técnica de conservación de bandeletas laterales prostáticas.

Los ejercicios del suelo pélvico constituyen un tratamiento no invasivo, de fácil uso y prácticamente libres de efectos adversos. Un programa de rehabilitación del suelo pélvico iniciado en el postoperatorio precoz, si bien no parece mejorar las tasas finales de continencia, ayuda a adelantar la recuperación de la misma y con ello a mejorar la calidad de vida de estos pacientes durante el postoperatorio.

\section{REFERENCIAS}

1. Kielb S, Dunn RL, Rashid MG, Sanda MG, Montie JE, Wei JT. Assessment of early continence recovery after radical prostatectomy: patient reported symptoms and impairment. J Urol. 2001 Sep;166(3):958-961.

2. Wei JT, Dunn RL, Marcovich R, Montie JE, Sanda MG. Prospective assessment of patient reported urinary continence after radical prostatectomy. J Urol. 2000 Sep;164(3 Pt 1):744-748.

3. Licht MR, Klein EA, Tuason L, Levin H. Impact of bladder neck preservation during radical prostatectomy on continence and cancer control. Urology. 1994 Dec;44(6):883-887.

4. Kundu SD, Roehl KA, Eggener SE, Antenor JA, Han M, Catalona WJ. Potency, continence and complications in 3,477 consecutive radical retropubic prostatectomies. J Urol. 2004 Dec;172(6 Pt 1):2227-2231.

5. Walsh PC, Marschke PL. Intussusception of the reconstructed bladder neck leads to earlier continence after radical prostatectomy. Urology. 2002 Jun;59(6):934-938.

6. Coakley FV, Eberhardt S, Kattan MW, Wei DC, Scardino PT, Hricak H. Urinary continence after radical retropubic prostatectomy: relationship with membranous urethral length on preoperative endorectal magnetic resonance imaging. J Urol. 2002 Sep; 168(3):1032-1035.

7. Gaker DL, Steel BL. Radical prostatectomy with preservation of urinary continence: pathology and long-term results. J Urol. 2004 Dec;172(6 Pt 2):2549-2552.

8. Maffezzini M, Seveso M, Taverna G, Giusti G, Benetti A, Graziotti P. Evaluation of complications and results in a contemporary series of 300 consecutive radical retropubic prostatectomies with the anatomic approach at a single institution. Urology. 2003 May;61(5):982-986. 
9. Poon M, Ruckle H, Bamshad BR, Tsai C, Webster R, Lui P. Radical retropubic prostatectomy: bladder neck preservation versus reconstruction. J Urol. 2000 Jan;163(1):194-198.

10. Filocamo MT, Li Marzi V, Del Popolo G. Effectiveness of early pelvic floor rehabilitation treatment for post-prostatectomy incontinence. Eur Urol. 2005 Nov;48(5):734-738.

11. Cornel EB, de Wit R, Witjes JA. Evaluation of early pelvic floor physiotherapy on the duration and degree of urinary incontinence after radical retropubic prostatectomy in a nonteaching hospital. World J Urol. 2005 Nov;23(5):353-355.

12. Queipo Zaragozá JA, Chicote Pérez F, Borrell Palanca A, Beltrán Meseguer JF, García Reboll L, Pastor Sempere F. Treatment of males with post-prostatectomy stress urinary incontinence by a mesh anchored to the ischio-pubic bones. Actas Urol Esp. 2005 Sep;29(8):764-768.

13. Haab F, Trockman BA, Zimmern PE, Leach GE. Quality of life and continence assessment of the artificial urinary sphincter in men with minimum 3.5 years of followup. $\mathrm{J}$ Urol. 1997 Aug; 158(2):435-439.

14. Sousa-Escandón A, Rodríguez Gómez JI, Uribarri González C, Marqués-Queimadelos A. Externally readjustable sling for treatment of male stress urinary incontinence: points of technique and preliminary results. J Endourol. 2004 Feb; 18(1):113-118.
15. Moore KN, Griffiths D, Hughton A. Urinary incontinence after radical prostatectomy: a randomized controlled trial comparing pelvic muscle exercises with or without electrical stimulation. BJU Int. 1999 Jan;83(1):57-65.

16. Van Kampen M, De Weerdt W, Van Poppel H, De Ridder D, Feys H, Baert L. Effect of pelvic-floor re-education on duration and degree of incontinence after radical prostatectomy: a randomised controlled trial. Lancet. 2000 Jan 8; 355(9198):98-102.

17. Wille S, Sobottka A, Heidenreich A, Hofmann R. Pelvic floor exercises, electrical stimulation and biofeedback after radical prostatectomy: results of a prospective randomized trial. J Urol. 2003 Aug; 170(2 Pt 1):490-493.

Dr. J.U. Juan Escudero

e-mail: chimojuan@hotmail.com

(Trabajo recibido el 10 de julio 2006) 